\title{
Eigenvalues of the Schrodinger equation for a periodic potential with nonperiodic boundary conditions: A uniform semiclassical analysis
}

\author{
J. N. L. Connor, ${ }^{\text {a) }}$ T. Uzer, ${ }^{\text {b) }}$ and R. A. Marcus \\ A. D. Smith ${ }^{\text {) }}$ \\ Department of Chemistry, University of Manchester, Manchester M13 9PL, England
}

A. A. Noyes Laboratory of Chemical Physics, ") California Institute of Technology, Pasadena, California 91125

(Received 8 September 1983; accepted 14 October 1983)

\begin{abstract}
A uniform semiclassical expression for the eigenvalues of a one dimensional periodic Schrödinger equation with nonperiodic boundary conditions has been derived. The potential energy function can have any number of symmetric or asymmetric barriers and wells. The treatment is uniform in that the classical turning points can come close together, coalesce, and move into the complex plane as the energy passes through a barrier maximum. A detailed application is made to Mathieu functions of integer order; the equations themselves include the case of fractional order.

Approximate semiclassical expressions are derived for the widths of the energy bands and the energy gaps of the periodic Mathieu equation when these quantities are small. The semiclassical results give a physical interpretation to formulas present in the mathematical literature and to the decrease in the splitting of a sequence of avoided crossings with increasing quantum numbers in coupled oscillator systems. Numerical calculations are reported to illustrate the high accuracy of the semiclassical formulas.
\end{abstract}

\section{INTRODUCTION}

The purpose of this article is to use uniform semiclassical techniques to study the eigenvalues of the one dimensional Schrödinger equation:

$$
\left[\frac{\hbar^{2}}{2 m} \frac{d^{2}}{d \theta^{2}}+E-V(\theta)\right] \Psi(\theta)=0
$$

when the potential energy function $V(\theta)$ is a periodic, analytic function of $\theta$, with period $2 \pi$ :

$$
V(\theta+2 \pi)=V(\theta) \text {. }
$$

The boundary condition for the wave function is taken in the form

$$
\Psi(\theta+2 \pi)=\exp (2 \pi \sigma i) \Psi(\theta),
$$

where $\sigma$ is real. Note that although $V(\theta)$ is periodic, the boundary condition for $\Psi(\theta)$ is nonperiodic, unless $\exp (2 \pi \sigma i)=1$.

The existence of a wave function satisfying the boundary condition (1.3) is a general result that follows from an application of Floquet's theorem to Hill's equation, ${ }^{1-7}$ of which the one dimensional Schrödinger equation with a periodic potential is a special case. For Eq. (1.1), Floquet's theorem states that there exists a particular solution which can be written in the product form ${ }^{1-7}$

$$
\Psi(\theta)=\exp (i \sigma \theta) P(\theta),
$$

where $P(\theta+2 \pi)=P(\theta)$ is periodic in $\theta$ and $\sigma$ is either real or complex. The boundary condition (1.3) then follows immedi-

\footnotetext{
a) Permanent address: Department of Chemistry, University of Manchester, Manchester M13 9PL, England.

b) Present address: Department of Chemistry, University of Colorado, Boulder, Colorado 80309.

c) Contribution number 6906.

d) Present address: Department of Physics, University of Waterloo, Waterloo, Ontario, N2L 3G1, Canada.
}

ately from Eq. (1.4). We also require that the wave function $\Psi(\theta)$ is always finite along the $\theta$ axis. This restricts $\sigma$ to real values and gives rise to gaps in the eigenvalue spectrum.

There are a number of areas in chemical physics that require a uniform semiclassical solution of Eq. (1.1) subject to the nonperiodic boundary condition (1.3):

(a) In the theory of coupled anharmonic oscillators, avoided crossings can occur when eigenvalues are plotted as a function of a perturbation parameter that couples the vibrations. $^{8-11}$ The use of Birkhoff-Gustavson classical perturbation theory together with a semiclassical quantization of the resulting good action-angle variables leads ${ }^{11}$ to an eigenvalue problem of the type Eqs. (1.1)-(1.3). Other theoretical treatments of coupled oscillator systems also result in similar eigenvalue problems. ${ }^{12,13}$

(b) In molecular spectroscopy, the theory of internal rotation $^{14-18}$ requires the solution of $\mathrm{Eq}$. (1.1) with the nonperiodic boundary condition (1.3). In particular, in the "internal axis method," coordinates are chosen to minimize or remove the coupling in the kinetic energy operator between the internal and overall rotation. ${ }^{14-18}$ The coupling still exists however and is apparent in the boundary condition (1.3). In the alternative "principal axis method," periodic solutions of Eq. (1.1) are required. ${ }^{16-18}$ Other areas of molecular spectroscopy in which Eqs. (1.1)-(1.3) occur are the theory of nonadiabatic transitions ${ }^{19}$ and Jahn-Teller molecules. ${ }^{20}$

(c) The boundary condition (1.3) is also fundamental in the band theory of solids where $\sigma$ is usually called the wave number and Eq. (1.4) represents a Bloch wave. ${ }^{3}$ In band theory, the main problem is to determine the range of $E$ for which $\sigma$ is real. These form the energy bands; they are separated by the energy gaps where $\sigma$ is complex.

In Sec. II we derive a uniform expression for the eigenvalues of Eq. (1.1) with the boundary condition (1.3) for a potential with $m$ symmetric or asymmetric barriers and 
wells. The treatment is uniform in the sense that the classical turning points can come close together and coalesce as the energy approaches the top of a barrier. ${ }^{21-23}$ For energies above the barrier maximum, the turning points move into the complex $\theta$ plane. This is possible because of our assumption that $V(\theta)$ is analytic in a region around the real $\theta$ axis. Although various special aspects of Eqs. (1.1)-(1.3) have been investigated previously by semiclassical techniques, ${ }^{1920,24-28}$ the general case has not been treated before and the present article therefore fills a gap in the literature.

In Sec. III we apply the results of Sec. II to the special case of $V(\theta)=2 q \cos 2 \theta$ with the boundary condition $\Psi(\theta+2 \pi)=\Psi(\theta)$, for which Eq. (1.1) reduces to Mathieu's equation. ${ }^{4,29-33}$ We derive several approximate formulas for the widths of the energy gaps and the energy bands when these quantities are small. In Sec. IV we present numerical tests of the semiclassical formulas.

We shall be particularly interested in the case of small energy gaps since in a recent theory of avoided crossings of eigenvalues in coupled oscillator systems, the energy gaps for the periodic Mathieu equation determine the minimum separation of these avoided crossings. ${ }^{11}$ In addition, the semiclassical theory allows a physical interpretation in terms of action integrals for the splitting of the eigenvalues. We also discuss the concept of "dynamical tunneling" which has been proposed by Davis and Heller ${ }^{34,35}$ to explain nearly degenerate eigenvalues in coupled oscillator systems. We also give a physical interpretation to some formulas for the widths of the energy gaps and bands of the Mathieu equation that have been reported in the mathematical literature.

Our conclusions are in Sec. V.

\section{UNIFORM SEMICLASSICAL THEORY}

\section{A. Introduction}

In this section we derive a uniform semiclassical expression for the eigenvalues of the Schrödinger equation (1.1) subject to the nonperiodic boundary condition (1.3).

We assume that $V(\theta)$ has $m$ maxima and minima in the range $(0,2 \pi]$. If instead $V(\theta)$ has $m$ maxima and minima in $(0, a]$ for $a>0$, then a simple linear transformation will transform the range to $(0,2 \pi]$ if necessary. The first two terms of the Fourier expansion of $V(\theta)$ may have the following form for example:

$$
V(\theta)=\frac{1}{2} V_{m}[1-\cos (m \theta)] .
$$

The potential (2.1) has $m$ symmetric barriers and wells in the range $(0,2 \pi]$, although our theory can also handle the case of asymmetric barriers and wells. As an example, Fig. 1 shows a plot of a symmetric threefold potential with the energy lying below the barrier maxima.

The derivation presented below is an improved version of one given sometime ago ${ }^{36}$ but not published then. The use of matrix techniques leads to an elegant solution of the problem. ${ }^{24,37}$ Our derivation is similar to that of Miller $^{24}$ except that we include a "quantum correction function" $\phi(\epsilon)$ in the semiclassical analysis and explicitly discuss the role of Stokes' points when the energy lies above a barrier maximum. In Sec. III we will derive formulas in which it is essential to include $\phi$ in the semiclassical analysis in order to avoid an infinite divergence at a barrier maximum.

\section{B. Uniform semiclassical wave functions and eigenvalues}

Figure 1 shows there are four classically allowed regions (denoted I-IV), separated by three identical symmetric barriers. In each classically allowed region a certain linear combination of semiclassical wave functions is valid. ${ }^{37}$ In region I we can write

$$
\begin{array}{r}
\Psi_{\mathrm{I}}(\theta) \sim \frac{\gamma_{1}}{k(\theta)^{1 / 2}} \exp \left[-i \int_{\theta}^{a_{1}} k\left(\theta^{\prime}\right) d \theta^{\prime}\right] \\
+\frac{\gamma_{2}}{k(\theta)^{1 / 2}} \exp \left[i \int_{\theta}^{a_{1}} k\left(\theta^{\prime}\right) d \theta^{\prime}\right]
\end{array}
$$

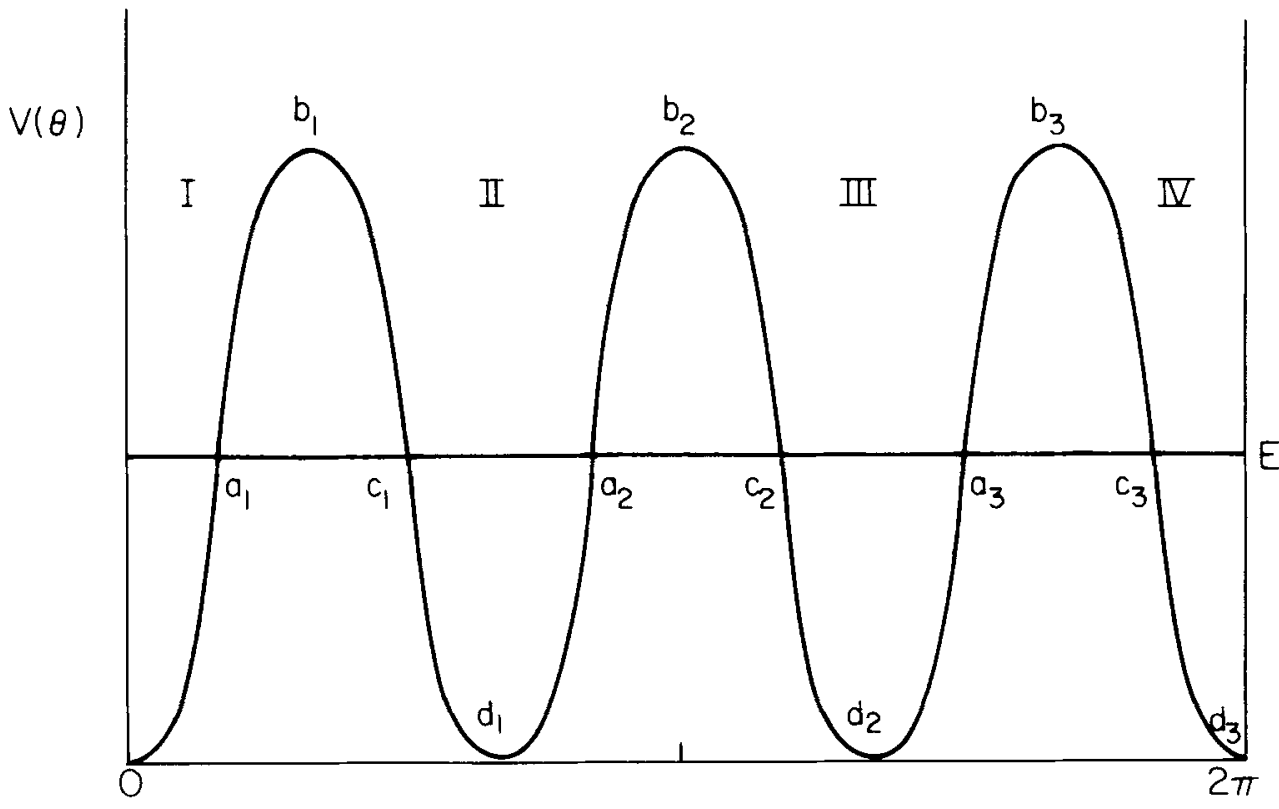

FIG. 1. Potential energy function $V(\theta)$ plotted against $\theta$ for the symmetrical threefold case. The classical turning points are denoted by $a_{1}, c_{1}, a_{2}, c_{2}, a_{3}, c_{3}$. The positions of the barrier maxima are denoted $b_{1}, b_{2}, b_{3}$ and the positions of the potential minima $d_{1}, d_{2}, d_{3}$. 
and in region II,

$$
\begin{aligned}
\Psi_{\mathrm{II}}(\theta) \sim & \frac{\gamma_{4}}{k(\theta)^{1 / 2}} \exp \left[-i \int_{c_{1}}^{\theta} k\left(\theta^{\prime}\right) d \theta^{\prime}\right] \\
& +\frac{\gamma_{3}}{k(\theta)^{1 / 2}} \exp \left[i \int_{c_{1}}^{\theta} k\left(\theta^{\prime}\right) d \theta^{\prime}\right],
\end{aligned}
$$

where

$$
k(\theta)=\{2 m[E-V(\theta)]\}^{1 / 2} / \hbar
$$

is the local wave number.
The first problem in the semiclassical theory is to connect the coefficients $\gamma_{1}$ and $\gamma_{2}$ in region I with the coefficients $\gamma_{3}$ and $\gamma_{4}$ in region II. To do this we use semiclassical parabolic connection formulas for a barrier, ${ }^{21-23}$ because the resulting wave function is then uniformly valid as the energy crosses the barrier maxima.

\section{If we write}

$$
\left(\begin{array}{l}
\gamma_{3} \\
\gamma_{4}
\end{array}\right)=\mathbf{C}\left(\begin{array}{l}
\gamma_{1} \\
\gamma_{2}
\end{array}\right)
$$

then the connection matrix $\mathbf{C}$ is given by ${ }^{21-23}$

$$
\mathbf{C}=\left(\begin{array}{cc}
{[1+\exp (-2 \pi \epsilon)]^{1 / 2} \exp (-i \phi)} & -i \exp (-\pi \epsilon) \\
i \exp (-\pi \epsilon) & {[1+\exp (-2 \pi \epsilon)]^{1 / 2} \exp (i \phi)}
\end{array}\right)
$$

where $\pi \epsilon$ is the action integral for the barrier

$$
\begin{aligned}
\pi \epsilon & =-i \int_{a_{1}}^{c_{1}} k(\theta) d \theta \\
& =-\int_{a_{1}}^{c_{1}}|k(\theta)| d \theta \text { when } V\left(d_{1}\right)<E<V\left(b_{1}\right),
\end{aligned}
$$

and $\phi(\epsilon)$ is the quantum correction function

$$
\left.\begin{array}{rl}
\phi(\epsilon) & =\epsilon+\arg \Gamma\left(\frac{1}{2}+i \epsilon\right)-\epsilon \ln |\epsilon| \\
& =-\phi(-\epsilon) .
\end{array}\right\}
$$

The branch of $k(\theta)$ in Eq. (2.7) is chosen so that $\epsilon<0$ when $E<V\left(b_{1}\right)$, where $b_{1}$ is the position of the barrier maximum. In Eqs. (2.2) and (2.3), the branch of $k(\theta)$ is selected so that the action integrals are always positive. In Eq. (2.9) the branch of $\arg \Gamma\left(\frac{1}{2}+i \epsilon\right)$ is not necessarily the principal one rather it is chosen so that $\phi(\epsilon) \rightarrow 0$ as $\epsilon \rightarrow \pm \infty$ with the requirement that $\phi(\epsilon)$ is a continuous function of $\epsilon$.

The next step is to write the action integrals in Eq. (2.3) in terms of the turning point $a_{2}$ associated with the second barrier in Fig. 1. We have

$$
\begin{aligned}
\Psi_{\mathrm{II}}(\theta) \sim & \frac{\gamma_{4}^{\prime}}{k(\theta)^{1 / 2}} \exp \left[i \int_{\theta}^{a_{2}} k\left(\theta^{\prime}\right) d \theta^{\prime}\right] \\
& +\frac{\gamma_{3}^{\prime}}{k(\theta)^{1 / 2}} \exp \left[-i \int_{\theta}^{a_{2}} k\left(\theta^{\prime}\right) d \theta^{\prime}\right],
\end{aligned}
$$

where

$$
\left(\begin{array}{l}
\gamma_{3}^{\prime} \\
\gamma_{4}^{\prime}
\end{array}\right)=\mathbf{B}\left(\begin{array}{l}
\gamma_{3} \\
\gamma_{4}
\end{array}\right)
$$

with

$$
\mathbf{B}=\left(\begin{array}{cc}
e^{i \alpha} & 0 \\
0 & e^{-i \alpha}
\end{array}\right)
$$

and

$$
\alpha=\int_{c_{1}}^{a_{2}} k(\theta) d \theta
$$

is the action integral for the well.

Next we use the connection equations (2.5) and (2.6) to find the form of the semiclassical wave function in region III; a second application of this procedure then gives us the wave function in region IV. If we write

$$
\begin{aligned}
\Psi_{\mathrm{IV}}(\theta) \sim & \frac{\gamma_{8}}{k(\theta)^{1 / 2}} \exp \left[-i \int_{c_{3}}^{\theta} k\left(\theta^{\prime}\right) d \theta^{\prime}\right] \\
& +\frac{\gamma_{7}}{k(\theta)^{1 / 2}} \exp \left[i \int_{c_{3}}^{\theta} k\left(\theta^{\prime}\right) d \theta^{\prime}\right],
\end{aligned}
$$

then we find $\gamma_{7}$ and $\gamma_{8}$ are related to $\gamma_{1}$ and $\gamma_{2}$ by

$$
\left(\begin{array}{l}
\gamma_{7} \\
\gamma_{8}
\end{array}\right)=\mathbf{C B C B C}\left(\begin{array}{l}
\gamma_{1} \\
\gamma_{2}
\end{array}\right)
$$

Next we impose the boundary condition

$$
\Psi(2 \pi)=\exp (2 \pi \sigma i) \Psi(0)
$$

on the semiclassical wave functions (2.2) and (2.14) and deduce that the boundary condition can be written in the form

$$
\left(\begin{array}{l}
\gamma_{7} \\
\gamma_{8}
\end{array}\right)=\exp (2 \pi \sigma i) \mathbf{B}^{-1}\left(\begin{array}{l}
\gamma_{1} \\
\gamma_{2}
\end{array}\right)
$$

which becomes

$$
\left[\mathbf{C B C B C}-\exp (2 \pi \sigma i) \mathbf{B}^{-1}\right]\left(\begin{array}{l}
\gamma_{1} \\
\gamma_{2}
\end{array}\right)=0
$$

using Eq. (2.15).

Equation (2.18) has nontrivial solutions for $\gamma_{1}$ and $\gamma_{2}$ only if

$$
\operatorname{det}\left|\mathbf{C B C B C}-\exp (2 \pi \sigma i) \mathbf{B}^{-1}\right|=0,
$$

which can be written in the alternative form

$$
\operatorname{det}\left|(\mathbf{C B})^{3}-\exp (2 \pi \sigma i) \mathbf{1}\right|=0
$$

since $\operatorname{det}|\mathbf{B}|=1$.

Equation (2.20) determines the eigenvalues for the threefold symmetric potential in Fig. 1. However the derivation does not require that each barrier be symmetric about its maximum and Eq. (2.20) is also valid for asymmetric barriers provided the potential still has a periodicity of $2 \pi / 3$.

For a potential of periodicity $2 \pi / m$ the generalization of Eq. $(2.20)$ is

$$
\operatorname{det}\left|(\mathbf{C B})^{m}-\exp (2 \pi \sigma i) \mathbf{1}\right|=0 .
$$

If the potential has $m$ different barriers and wells but has only a periodicity of $2 \pi$, then the eigenvalue equation is

$$
\operatorname{det}\left|(\mathbf{C B})_{1}(\mathbf{C B})_{2} \ldots(\mathbf{C B})_{m}-\exp (2 \pi \sigma i) \mathbf{1}\right|=0
$$

with a different $\epsilon_{i}, \alpha_{i}$ for each barrier and well, respectively.

The derivation so far has been restricted to cases where the energy lies below the maxima of all the barriers. How- 


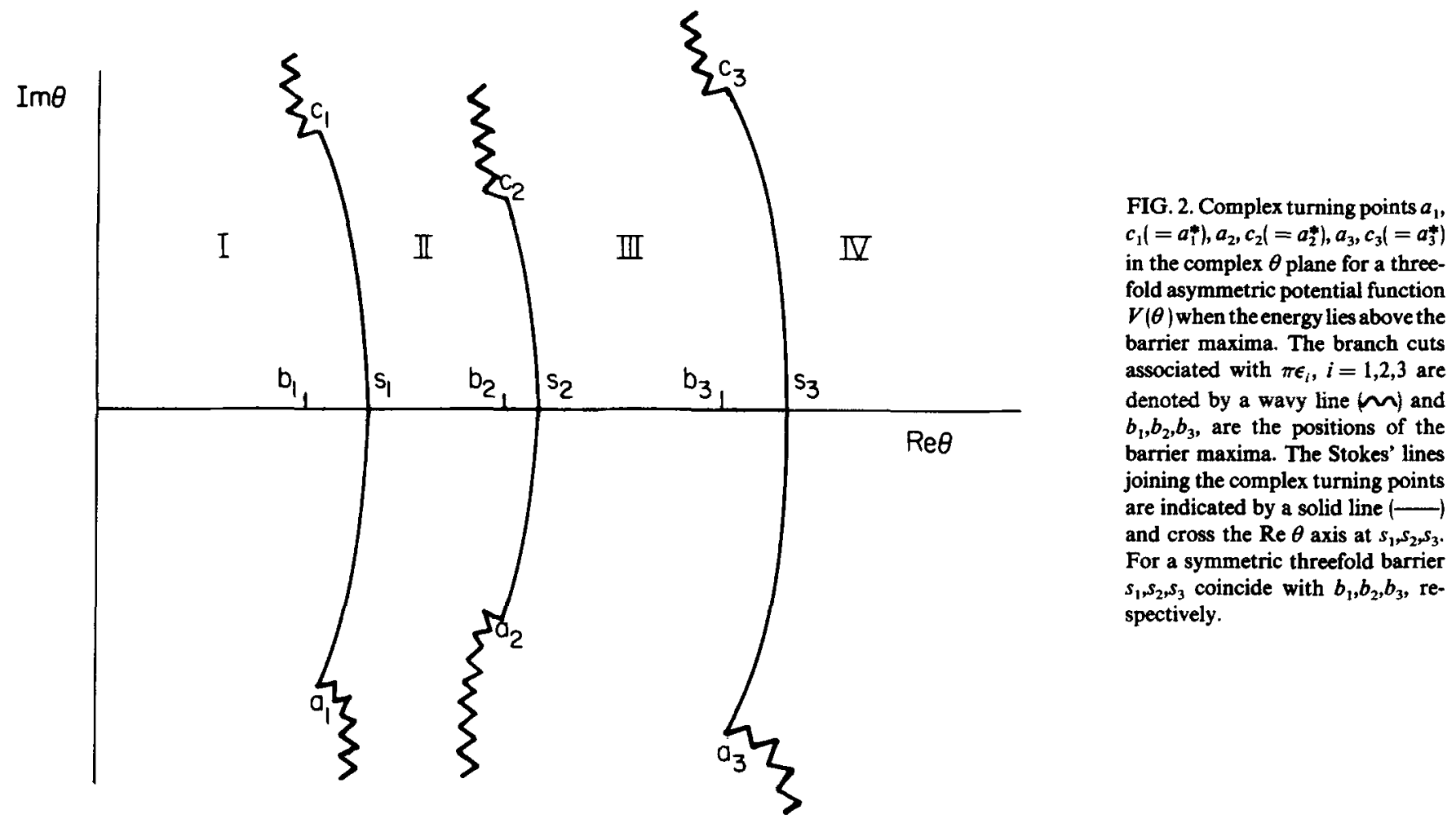

ever, the derived formulas remain valid when the energy is above the maxima of some or all of the barriers, provided we make certain modifications.

Consider the $m=3$ fold symmetric potential in Fig. 1 again. When $E$ lies above $V\left(b_{1}\right)$, the turning points move into the complex $\theta$ plane with $a_{1}=c_{1}^{*}$. The definition (2.7) for $\pi \epsilon$ remains valid with the branch of $k(\theta)$ chosen so that $\epsilon>0$. For the action integral $\alpha$, the integration limits are now the Stokes' points $s_{1}$ and $s_{2}$ (see Fig. 2). However for a symmetric barrier $s_{1}$ coincides with $b_{1}$ the position of the barrier maximum, and we can write (see Fig. 1).

$$
\alpha=\int_{b_{1}}^{b_{2}} k(\theta) d \theta, \quad E>V\left(b_{1}\right) \text { (symmetric barrier) . }
$$

For an asymmetric barrier, this is no longer true and we must write instead (see Fig. 2)

$$
\alpha_{1}=\int_{s_{1}}^{s_{2}} k(\theta) d \theta, \quad E>V\left(b_{1}\right), \quad \text { and } E>V\left(b_{2}\right) \text {. }
$$

In applications, Eq. (2.24) requires the numerical evaluation of $s_{1}$ and $s_{2}$. To avoid having to calculate these quantities, it is convenient to exploit the fact that, by definition, ${ }^{26,41}$ the action integral is pure imaginary along a Stokes' line; $\alpha_{1}$ can then be calculated from

$$
\alpha_{1}=\operatorname{Re} \int_{c_{1}}^{a_{2}} k(\theta) d \theta, \quad E \xi V\left(b_{1}\right), \quad \text { and } E \gtrless V\left(b_{2}\right) .
$$

Equation (2.25) is also valid when one of the turning points is real and the other one is complex. This situation occurs for example if $E<V\left(b_{1}\right)$ and $E>V\left(b_{2}\right)$.

It is also necessary to remember that the general formula (2.22) assumes that parabolic connection formulas ${ }^{21-23}$ can be applied to each barrier at a given eigenenergy. In some applications, the minimum of one well may lie above the maximum of one or more of the other barriers. In this circumstance, Eq. (2.22) may not be valid and it may be necessary to use a different kind of connection formula in the analysis. For example, parabolic connection formulas for a well may be appropriate ${ }^{21.38}$ if it is necessary to account for the coalescence of the turning points associated with the well.

\section{Uniform semiclassical eigenvalues for a potential of periodicity $2 \pi / m$}

The formula (2.22) for the energy eigenvalues of a general asymmetric potential cannot be further simplified. When the potential possesses some symmetry however, additional simplifications may be possible. We shall now consider the case of a potential of periodicity $2 \pi / m$ as this case often arises in applications.

Since $\operatorname{det}|\mathbf{C}|=\operatorname{det}|\mathbf{B}|=1$, it is straightforward to deduce that $\operatorname{det}\left|(\mathbf{C B})^{m}\right|=1$, and that Eq. (2.21) is equivalent to

$$
\operatorname{Tr}\left[(\mathbf{C B})^{m}\right]=2 \cos (2 \pi \sigma),
$$

where Tr denotes the trace of a matrix. Now a trace is invariant under a similarity transformation $\mathbf{U}$ :

$$
\operatorname{Tr}\left[(\mathbf{C B})^{m}\right]=\operatorname{Tr}\left[\left(\mathbf{U C B U}^{-1}\right)^{m}\right],
$$

and if $\mathbf{U}$ is chosen so that $\mathrm{UCBU}^{-1}$ is diagonal, then the diagonal elements of $\mathbf{U C B U}^{-1}$ are equal to the eigenvalues of CB. To find the eigenvalues of $\mathrm{CB}$, we solve the equation

$$
\operatorname{det}|\mathbf{C B}-\lambda \mathbf{1}|=0 \text {, }
$$

obtaining from the characteristic equation for $\lambda$ the eigenvalues

$$
\lambda_{ \pm}=\exp ( \pm i \chi)
$$


where

$$
\cos \chi=[1+\exp (-2 \pi \epsilon)]^{1 / 2} \cos (\alpha-\phi) .
$$

We then have

$$
\operatorname{Tr}\left[(\mathbf{C B})^{m}\right]=\lambda_{+}^{m}+\lambda_{-}^{m}
$$

or using Eq. (2.29):

$$
\operatorname{Tr}\left[(\mathrm{CB})^{m}\right]=2 \cos (m \chi) \text {. }
$$

Finally, equating Eqs. (2.26) and (2.32), we obtain the uniform semiclassical quantization condition for a potential of periodicity $2 \pi / m$ :

$$
[1+\exp (-2 \pi \epsilon)]^{1 / 2} \cos (\alpha-\phi)=\cos [2 \pi(j-\sigma) / m],
$$

where $j=0, \pm 1, \pm 2, \ldots$. Equation (2.33) is the main result of this subsection.

For special values of $\sigma$ and $m$, the general result (2.33) reduces to formulas that have been reported in the literature. For $m=3$ and $\sigma=\frac{1}{2}$ [so that $\Psi(\theta+2 \pi)=-\Psi(\theta)$ ], Eq. (2.33) is equivalent to the result of Karkach and Osherov [Eq. (35) of Ref. 20]. Karkach et al. ${ }^{19}$ have also studied the case $\sigma=\frac{1}{2}$ for the potential $V(\theta)=(\beta+\cos 2 \theta)^{2}$ and for energies near the the maxima of the four barriers of $V(\theta)$, their quantization condition [Eq. (26) of Ref. 19] is a special case of the more general formual (2.22). When $m=3$ and $\sigma=0$, Eq. (2.33) reduces to the equation used in Ref. 25 to study the torsional vibrational energy levels of acetaldehyde. For $m=2$ and $\sigma=0$, Eq. (2.33) is equivalent to the semiclassical quantization condition applied in Ref. 39 to the energy levels of a rigid asymmetric rotor. Finally when $m=1$, so that the potential has a single barrier per $2 \pi$, we obtain the first order dispersion formula derived by Fröman [Eq. (40) of Ref. 26] using phase integral methods.

Various other more approximate formulas in the literature ${ }^{12,13,24,40}$ can be obtained from Eq. (2.33) by setting $\phi \equiv 0$. However, the definition of $\phi(\epsilon)$ [Eq. (2.9)] shows that $\phi(\epsilon)$ is not identically zero except for $\epsilon=0$ and $\epsilon= \pm \infty$. The quantum correction function $\phi(\epsilon)$ is important for energies near the top of the barrier because it cancels a logarithmic singularity in the action integral $\alpha .^{22,41}$

It is interesting to note that in the related problems of orbiting collisions in elastic scattering $38,41,42$ and for the eigenvalues of a double well potential, ${ }^{43} \phi$ is related to the action integral $\alpha$ in the combination $\alpha-\frac{1}{2} \phi$ rather than $\alpha-\phi$ as is the case here. This illustrates that it is not straightforward to "guess" the quantization rule $(2.33)$ by examining results for related problems or even by considering formulas for special values of $m$ and $\sigma$ which have $\phi \equiv 0$. The reason why $\alpha-\phi$ occurs in Eq. (2.33) is that $\alpha$ receives singular contributions from two barrier maxima unlike the orbiting and double well problems where just a single barrier maximum contributes to the singularity in $\alpha$.

\section{EXAMPLE: THE MATHIEU EQUATION}

\section{A. Introduction}

In this section we apply the general uniform semiclassical quantization formula (2.33) to the special case of Mathieu's equation. Mathieu's equation is obtained from Eq. (1.1) by setting $\hbar=1, m=\frac{1}{2}$ and choosing $V(\theta)=2 q \cos 2 \theta$ with $q>0$ so that $V(\theta)$ has two barriers in $(0,2 \pi]$. We then have

$$
\frac{d^{2} \Psi}{d \theta^{2}}+(a-2 q \cos 2 \theta) \Psi=0,
$$

where $E$ has also been replaced by $a$ so as to obtain the canonical form of Mathieu's equation. ${ }^{29}$ The boundary condition is taken to be

$$
\Psi(\theta+2 \pi)=\Psi(\theta) .
$$

Solutions of Eq. (3.1) with this boundary condition are usually denoted $c e_{n}(\theta), n=0,1,2, \ldots$ and $\operatorname{se}_{n}(\theta), n=1,2,3, \ldots$ with eigenvalues $a_{n}$ and $b_{n}$, respectively. ${ }^{29}$ Note that we could have used the alternative boundary condition

$$
\Psi(\theta+\pi)= \pm \Psi(\theta)
$$

for which the Mathieu potential has one barrier per period $\pi$.

Physically, Mathieu's equation corresponds to a plane pendulum in quantum mechanics. ${ }^{44}$ With the more general boundary condition (1.3), it also arises in many other different areas of physics and chemistry including: theories of avoided crossings ${ }^{11}$ and local mode behavior ${ }^{12}$ of coupled oscillator systems (see also Ref. 45), band theory, $3,26-28,46$ problems involving hindered rotations, ${ }^{14-18,47}$ field theory, ${ }^{48}$ soliton free energies, ${ }^{49}$ atoms and molecules in external fields, ${ }^{50}$ as well as in other problems. ${ }^{51}$

Mathematically, the Mathieu equation has been well studied. ${ }^{29-33}$ Nevertheless, there are still interesting features to be learned from it as we demonstrate below. In particular for the boundary condition (3.2) we derive semiclassical formulas for the widths of the energy gaps (corresponding to $a$ / $q>1)$ and for the widths of the energy bands $(a / q<1)$ when both these quantities are small. This also allows us to give a physical interpretation to some formulas in the mathematical literature. In addition, we present in Sec. IV a numerical test of the accuracy of the various semiclassical formulas.

\section{B. Semiclassical theory}

$$
\begin{aligned}
& \text { Setting } m=2, \sigma=0 \text { in Eq. (2.33) gives } \\
& \cos (\alpha-\phi)= \pm[1+\exp (-2 \pi \epsilon)]^{-1 / 2}
\end{aligned}
$$

as the uniform semiclassical quantization condition that determines the width of the energy bands (and hence the width of the energy gaps). Equation (3.3) can also be written in the alternative form ${ }^{26}$

$$
\begin{aligned}
& \alpha-\phi=\left(m-\frac{1}{2}\right) \pi \pm \tan ^{-1}[\exp (\pi \epsilon)], \\
& m=1,2,3, \ldots,
\end{aligned}
$$

where the plus (minus) sign determines the upper (lower) edge of the $m$ th band and the arctangent takes its principal value. In conventional Mathieu notation the width of the $m$ th band is obtained from $b_{m}-a_{m-1}$.

The $m$ th gap is defined as the difference between the energy of the lower edge of the $(m+1)$ th band and the upper edge of the $m$ th band. With the help of the identity

$$
\tan ^{-1}[\exp (-\pi \epsilon)]+\tan ^{-1}[\exp (\pi \epsilon)]=\pi / 2,
$$

we obtain

$$
\begin{aligned}
& \alpha-\phi=m \pi \pm \tan ^{-1}[\exp (-\pi \epsilon)], \\
& m=1,2,3, \ldots,
\end{aligned}
$$

where the plus (minus) sign determines the upper (lower) 
edge of the $m$ th gap. The width of the $m$ th gap is given by $a_{m}-b_{m}$ in conventional Mathieu notation.

Next we consider the case of narrow energy bands. Equation (3.4) shows that the energy of the center of the band is given approximately by ${ }^{26}$

$$
\alpha-\phi=\left(m-\frac{1}{2}\right) \pi, \quad m=1,2,3, \ldots .
$$

Equation (3.6) is a Bohr-Sommerfeld quantization condition, modified by the presence of the quantum correction function $\phi$. If the energy is well below the barrier maximum, $\phi$ can be neglected and we have simply

$$
\alpha=\left(m-\frac{1}{2}\right) \pi, \quad m=1,2,3, \ldots .
$$

We now expand $\alpha-\phi$ in Eq. (3.4) to first $\operatorname{order}^{22,38}$ about the energy of the center of the band, (which will be denoted ${ }^{b} \bar{E}_{m}$ ), obtaining for the width of the band ${ }^{b} \Delta_{m}$ :

$$
\begin{aligned}
& { }^{b} \Delta_{m}^{(1)}\left({ }^{b} \bar{E}_{m}\right)=\frac{2}{d \bar{\alpha} /\left.d E\right|_{E={ }^{b} \bar{E}_{m}}} \tan ^{-1}\left\{\exp \left[\pi \epsilon\left({ }^{b} \bar{E}_{m}\right)\right]\right\}, \\
& m=1,2,3, \ldots,
\end{aligned}
$$

where

$$
\bar{\alpha}=\alpha-\phi .
$$

Equation (3.8) is valid for energies below, at, or above the top of the barrier, provide the bandwidth remains narrow. At the barrier maximum $d \alpha / d E$ is infinite, but this singularity is exactly cancelled by one in $d \phi / d E$, with the result that $d \bar{\alpha} /$ $d E$ is finite. The quantity $d \phi / d E$ is related to the digamma function $\psi(z)$ by ${ }^{41}$

$$
\frac{d \phi[\epsilon(E)]}{d E}=\left[-\ln |\epsilon|+\operatorname{Re} \psi\left(\frac{1}{2}+i \epsilon\right)\right] \frac{d \epsilon}{d E} .
$$

If we approximate the $\tan ^{-1} x$ factor in Eq. (3.8) by the first term of its Taylor series expansion when $x<1$ we obtain

$$
\begin{aligned}
& { }^{b} \Delta_{m}^{(2)}\left({ }^{b} \bar{E}_{m}\right)=\frac{2}{d \bar{\alpha} /\left.d E\right|_{E={ }^{b} \bar{E}_{m}}} \exp \left[\pi \epsilon\left({ }^{b} \bar{E}_{m}\right)\right], \\
& m=1,2,3, \ldots
\end{aligned}
$$

When the energy of the center of the band lies well below the barrier maximum, we can neglect the $d \phi / d E$ term, obtaining from Eq. (3.8):

$$
\begin{aligned}
& { }^{b} \Delta_{m}^{(3)}\left({ }^{(} \bar{E}_{m}\right)=\frac{2 \hbar \omega\left({ }^{b} \bar{E}_{m}\right)}{\pi} \tan ^{-1}\left\{\exp \left[\pi \epsilon\left({ }^{b} \bar{E}_{m}\right)\right]\right\}, \\
& m=1,2,3, \ldots,
\end{aligned}
$$

where $d \alpha / d E=\pi / \hbar \omega$ and

$$
\omega(E)=\pi \hbar /\left[m \int_{c_{1}}^{a_{2}} \frac{d \theta}{k(\theta)}\right]
$$

is the classical angular frequency of oscillation between $c_{1}$ and $a_{2}\left(\right.$ or $c_{2}$ and $\left.a_{1}\right)$. If we again approximate the $\tan ^{-1}$ term in Eq. (3.12) we get

$$
\begin{aligned}
& { }^{b} \Delta{ }_{m}^{(4)}\left({ }^{b} \bar{E}_{m}\right)=\frac{2 \hbar \omega\left({ }^{b} \bar{E}_{m}\right)}{\pi} \exp \left[\pi \epsilon\left({ }^{b} \bar{E}_{m}\right)\right], \\
& m=1,2,3, \ldots .
\end{aligned}
$$

It is interesting to compare the bandwidth (3.14) with the semiclassical expression for the splitting of the energy levels of a symmetric double well potential, ${ }^{52}$ which is $(\hbar \omega /$ $\pi) \exp (\pi \epsilon)$. The extra factor of 2 in Eq. (3.14) can be understood physically in that the particle can tunnel in either di- rection for the Mathieu potential whereas in the double well problem it can only tunnel in one direction (namely to the other well).

We now derive a similar set of semiclassical formulas to those just given but for the case of narrow energy gaps. From Eq. (3.5), the energy of the center of the gap is given approximately by ${ }^{26}$

$$
\alpha-\phi=m \pi, \quad m=1,2,3, \ldots .
$$

This is a modified Bohr-Sommerfeld quantization condition analogous to Eq. (3.6). If we neglect $\phi$, which is permissible for energies well above the barrier maxima we obtain

$$
\alpha=m \pi, \quad m=1,2,3, \ldots .
$$

Notice that for the case of narrow energy bands, the quantization formulas (3.6) and (3.7) have $\left(m-\frac{1}{2}\right) \pi$ on their righthand sides, as is appropriate for vibrational motions, whereas for narrow energy gaps, $m \pi$ occurs, as is expected for rotational problems without real classical turning points.

Expanding $\alpha-\phi$ to first order about the energy of the center of the gap ${ }^{g} \bar{E}_{m}$, we then obtain for the gap width ${ }^{g} \Delta_{m}$ :

${ }^{8} \Delta_{m}^{(1)}\left({ }^{8} \bar{E}_{m}\right)=\frac{2}{d \bar{\alpha} /\left.d E\right|_{E={ }^{8} \bar{E}_{m}}} \tan ^{-1}\left\{\exp \left[-\pi \epsilon\left({ }^{8} \bar{E}_{m}\right)\right]\right\}$,

$m=1,2,3, \ldots$

and approximating the $\tan ^{-1}$ term gives

$$
\begin{aligned}
& \left.{ }^{g} \underset{m}{(2)}\left(g \bar{E}_{m}\right)=\frac{2}{d \bar{\alpha} /\left.d E\right|_{E={ }^{8} \bar{E}_{m}}} \exp \left[-\pi \epsilon{ }^{8} \bar{E}_{m}\right)\right], \\
& m=1,2,3, \ldots .
\end{aligned}
$$

Equations (3.17) and (3.18) are valid at the barrier maxima if the gap width is sufficiently narrow.

When ${ }^{8} \bar{E}_{m}$ is well above the barrier maxima so that $d \phi /$ $d E$ can be neglected, we obtain equations analogous to Eqs. (3.12) and (3.14), namely,

$$
\begin{aligned}
& { }^{s}{ }_{m}^{(3)}\left({ }^{(8} \bar{E}_{m}\right)=\frac{2 \hbar \omega\left({ }^{8} \bar{E}_{m}\right)}{\pi} \tan ^{-1}\left\{\exp \left[-\pi \epsilon\left({ }^{8} \bar{E}_{m}\right)\right]\right\}, \\
& m=1,2,3, \ldots
\end{aligned}
$$

and

$$
\begin{aligned}
& { }^{g} \Delta_{m}^{(4)}\left({ }^{(8} \bar{E}_{m}\right)=\frac{2 \hbar \omega\left({ }^{8} \bar{E}_{m}\right)}{\pi} \exp \left[-\pi \epsilon\left({ }^{8} \bar{E}_{m}\right)\right], \\
& m=1,2,3, \ldots,
\end{aligned}
$$

where

$$
\omega(E)=\pi \hbar /\left[m \int_{\theta}^{\theta+\pi} \frac{d \theta^{\prime}}{k\left(\theta^{\prime}\right)}\right] .
$$

Alternatively, Eqs. (3.19) and (3.20) can be written in terms of the time $t(E)$ for the particle to cross one period $\pi$ of the potential since $t(E)=\pi / \omega(E)$. Equations (3.16) and (3.20) are equivalent to the formulas derived by Dykhne. ${ }^{53}$ Notice that in the expressions for ${ }^{b} \Delta_{m}^{(i)}(E)$ and ${ }^{g} \Delta_{m}^{(i)}(E)$ it is necessary to specify the energy $E$ to be used for their evaluation. The derivation presented above corresponds to the choice $E={ }^{b} \bar{E}_{m}$ or $E={ }^{s} \bar{E}_{m}$.

The derivation given in this section has not used any specific properties of the Mathieu potential $V(\theta)=2 q \cos 2 \theta$; the equations are in fact valid for any po- 
tential with one barrier per period $\pi$. Another interesting point concerns Eq. (3.20) which shows the gap widths become exponentially small as ${ }^{8} \bar{E}_{m}$ increases. It is known that this is a general result ${ }^{54}$ which follows from our assumption that $V(\theta)$ is an analytic function of $\theta$ in a region around the $\operatorname{Re} \theta$ axis.

\section{Use of elliptic integrals in the semiclassical formulas for Mathieu's equation}

We now apply the semiclassical formulas derived in Sec. III B to the special case of Mathieu's equation (3.1). In particular we express the action integrals and their derivatives in terms of complete elliptic integrals of the first and second kinds $K(k)$ and $E(k)$, respectively, where $k$ is the modulus. We use the notation of Byrd and Friedman ${ }^{55}$ rather than the alternative notation of Milne-Thomson. ${ }^{56}$ By using elliptic integrals we can exploit the considerable body of theory that exists for these functions. ${ }^{55}$ In addition, it is easy to perform numerical calculations since efficient subroutines are available for the evaluation of $K(k)$ and $E(k)$ in most program libraries.

It is convenient in the numerical calculations to have the modulus $k$ of the elliptic integrals satisfy $0<k<1$. This requires that we distinguish between the case of real turning points $(-2 q \leqslant a \leqslant 2 q)$ and complex turning points $(a>2 q)$. Notice that when $a>2 q$, a convenient alternative form for the action integral $\alpha$ is

$$
\alpha=\int_{\theta}^{\theta+\pi} k\left(\theta^{\prime}\right) d \theta^{\prime},
$$

see also Eq. (3.21).

The derivation of $\alpha, \pi \epsilon, d \alpha / d a, d(\pi \epsilon) / d a$ in terms of $K(k)$ and $E(k)$ is generally straightforward ${ }^{26}$ so we just quote the final results.

(a) When $-2 q \leqslant a \leqslant 2 q$,

$$
\begin{aligned}
\alpha= & 4 q^{1 / 2}\left[E\left(\left(\frac{a+2 q}{4 q}\right)^{1 / 2}\right)\right. \\
& \left.+\left(\frac{a-2 q}{4 q}\right) K\left(\left(\frac{a+2 q}{4 q}\right)^{1 / 2}\right)\right], \\
\pi \epsilon= & -4 q^{1 / 2}\left[E\left(\left(\frac{2 q-a}{4 q}\right)^{1 / 2}\right)\right. \\
& \left.-\left(\frac{2 q+a}{4 q}\right) K\left(\left(\frac{2 q-a}{4 q}\right)^{1 / 2}\right)\right], \\
\frac{d \alpha}{d a}= & \frac{1}{2 q^{1 / 2}} K\left(\left(\frac{a+2 q}{4 q}\right)^{1 / 2}\right), \\
\frac{d(\pi \epsilon)}{d a}= & \frac{1}{2 q^{1 / 2}} K\left(\left(\frac{2 q-a}{4 q}\right)^{1 / 2}\right) .
\end{aligned}
$$

(b) When $a>2 q$,

$\alpha=2(a+2 q)^{1 / 2} E\left(\left(\frac{4 q}{a+2 q}\right)^{1 / 2}\right)$,

$\pi \epsilon=2(a+2 q)^{1 / 2}\left[K\left(\left(\frac{a-2 q}{a+2 q}\right)^{1 / 2}\right)-E\left(\left(\frac{a-2 q}{a+2 q}\right)^{1 / 2}\right)\right]$,

$$
\begin{aligned}
& \frac{d \alpha}{d a}=\frac{1}{(a+2 q)^{1 / 2}} K\left(\left(\frac{4 q}{a+2 q}\right)^{1 / 2}\right), \\
& \frac{d(\pi \epsilon)}{d a}=\frac{1}{(a+2 q)^{1 / 2}} K\left(\left(\frac{a-2 q}{a+2 q}\right)^{1 / 2}\right) .
\end{aligned}
$$

It can be checked using the identities in Ref. 55 together with the fact that $k$ is allowed to take any real or complex value that the equations for $a>2 q$ can be obtained directly from those for $-2 q \leqslant a \leqslant 2 q$.

\section{Narrow bandwidths and gaps for Mathieu's equation}

When $-2 q<a<2 q$ and $a / q<1$, the energy bands are very narrow and Eq. (3.14) is valid. Similarly for $a / q>1$ the energy gaps are very narrow and Eq. (3.20) applies. The existence of narrow energy bands and gaps is also readily apparent from an inspection of the stability diagram for Mathieu's equation. ${ }^{57,58}$ We now show that the semiclassical equations derived in Sec. III B are asymptotically equivalent to some results reported in the mathematical literature. This gives a physical interpretation to these mathematical results which apparently has not been done before.

First we consider Eqs. (3.7) and (3.23) for the energy of the center of a narrow band. If we write for the Bohr-Sommerfeld energy

$$
{ }^{b} \bar{a}_{m}^{B S}=-2 q+\delta_{m}, \quad m=1,2,3, \ldots,
$$

then we can expand $E(k)$ and $K(k)$ for small $k$ using the expansions $^{55}$

$$
\left.\begin{array}{l}
E(k)=\frac{1}{2} \pi\left(1-\frac{1}{4} k^{2}\right)+0\left(k^{4}\right), \\
K(k)=\frac{1}{2} \pi\left(1+\frac{1}{4} k^{2}\right)+0\left(k^{4}\right) .
\end{array}\right\}
$$

We then find that

$$
{ }^{b} \bar{a}_{m}^{B S} \sim-2 q+4\left(m-\frac{1}{2}\right) q^{1 / 2}, \quad m=1,2,3, \ldots
$$

or recognizing that $a_{m-1}, b_{m}$. and ${ }^{b} \bar{a}_{m}^{B S}$ are all asymptotically equal, since the bandwidth is exponentially small, we can write

$$
\begin{aligned}
& { }^{b} \bar{a}_{m}^{B S} \sim b_{m} \sim a_{m-1} \sim-2 q+4\left(m-\frac{1}{2}\right) q^{1 / 2}, \\
& m=1,2,3, \ldots .
\end{aligned}
$$

Equation (3.34) agrees with the rigorous mathematical result. ${ }^{59,60}$ Physically, the Bohr-Sommerfeld quantization condition (3.7) corresponds to vibrational motion in either of the two wells of the Mathieu potential and Eq. (3.34) can be recognized as the harmonic oscillator result.

Next we consider the semiclassical formula (3.14) for the width of a narrow band. If we substitute ${ }^{b} \bar{a}_{m}^{B S}$ $=-2 q+4\left(m-\frac{1}{2}\right) q^{1 / 2}$ into the integral $\pi \epsilon$, then we can use the following expansions ${ }^{55}$ in Eq. (3.24):

$$
\begin{aligned}
& E\left(k^{\prime}\right)=1+\frac{1}{2}\left[\ln (4 / k)-\frac{1}{2}\right] k^{2}+0\left(k^{4}\right), \\
& K\left(k^{\prime}\right)=\ln (4 / k)+\frac{1}{4}[\ln (4 / k)-1] k^{2}+0\left(k^{4}\right),
\end{aligned}
$$

where

$$
k^{\prime}=\left(1-k^{2}\right)^{1 / 2}
$$

is the complementary modulus. We then obtain for the asymptotic semiclassical bandwidth the formula 


$$
\begin{aligned}
& { }^{b}{ }_{m}^{(4) a s}=2^{4 m+1} \frac{\exp \left(m-\frac{1}{2}-4 q^{1 / 2}\right)}{\pi\left(m-\frac{1}{2}\right)^{m-\frac{1}{2}}} q^{\frac{1}{2 m+1}}, \\
& m=1,2,3, \ldots .
\end{aligned}
$$

Meixner and Schäfke ${ }^{61}$ and Blanch ${ }^{62}$ quote without proof the following rigorous asymptotic mathematical expression for the bandwidth:

$$
\begin{aligned}
& { }^{b} \Delta_{m}^{a s}=2^{4 m+1} \frac{2^{1 / 2} \exp \left(-4 q^{1 / 2}\right)}{\pi^{1 / 2}(m-1) !} q^{1 m+1}, \\
& m=1,2,3, \ldots .
\end{aligned}
$$

It should be noted however that Eq. (3.38) is actually due to Goldstein. ${ }^{63,64}$ If we use Stirling's form ${ }^{65}$ of Stirling's approximation

$$
\begin{aligned}
& (m-1) ! \sim(2 \pi)^{1 / 2}\left(m-\frac{1}{2}\right)^{m-1} \exp \left[-\left(m-\frac{1}{2}\right)\right] \\
& m=1,2,3, \ldots,
\end{aligned}
$$

we see that Eq. (3.37) is obtained. The approximation (3.39) is only in error by $7.5 \%$ for $m=1$ and $2.8 \%$ for $m=2$. By comparing Eqs. (3.37) and (3.38) we can improve the semiclassical expression ${ }^{b} \Delta{ }_{m}^{(4)}$ so that it gives the correct result for the Mathieu potential without having to invoke Stirling's approximation, namely,

$$
\begin{aligned}
& { }^{b}{ }_{m}^{(5)}\left({ }^{b} \bar{E}_{m}\right)=f_{m} \frac{2 \hbar \omega\left({ }^{b} \bar{E}_{m}\right)}{\pi} \exp \left[\pi \epsilon\left({ }^{b} \bar{E}_{m}\right)\right], \\
& m=1,2,3, \ldots,
\end{aligned}
$$

where

$$
f_{m}=\frac{(2 \pi)^{1 / 2}\left(m-\frac{1}{2}\right)^{m-\frac{1}{2}}}{e^{m-\frac{1}{2}(m-1) !}}, \quad m=1,2,3, \ldots .
$$

It is likely that the result (3.40) can be derived using semiclassical connection formulas for a parabolic well since the correction factor $f_{m}$ occurs naturally in that approach. ${ }^{21,38,66,67}$

We now consider the case of narrow energy gaps which occur for $a / q>1$. Notice that this inequality includes $q>1$ as well as the perturbation limit $q \rightarrow 0$.

The Bohr-Sommerfeld quantization condition (3.16) determines the energy ${ }^{8} \bar{a}_{m}^{B S}$, of the center of a gap. When $q=0$, Eq. (3.16) gives ${ }^{8} \bar{a}_{m}^{B S}=m^{2}$, which is the plane rotor result and Eq. (3.20) correctly yields ${ }^{8} \Delta{ }_{m}^{(4)} \equiv 0$, i.e., there are no gaps for $q=0$. It is known that $V(\theta) \equiv$ constant is the only potential for which this behavior occurs. ${ }^{68}$ Note that every energy level is doubly degenerate (except for the ground state level $a_{0}$ ) when $q=0$.

For $q \neq 0$, we use the approximation (3.32) for $E(k)$ in Eqs. (3.16) and (3.27) to obtain for the energy of the center of the gap

$$
{ }^{8} \bar{a}_{m}^{B S} \sim m^{2}+\frac{1}{2} q^{2} / m^{2}, \quad m=1,2,3, \ldots .
$$

The exact result for $m \geqslant 3$ is ${ }^{69}$

$$
a_{m} \sim b_{m} \sim m^{2}+\frac{1}{2} q^{2} /\left(m^{2}-1\right), \quad m=3,4,5, \ldots .
$$

Thus the semiclassical result makes an error in the denominator of the second term of Eq. (3.42) where it has $m^{2}$ instead of $m^{2}-1$.

In order to calculate the asymptotic form for the width of the energy gap ${ }^{8} \Delta_{m}^{(4)}$ we need to use the expansions (3.35) and (3.36) for $E\left(k^{\prime}\right)$ and $K\left(k^{\prime}\right)$ in Eq. (3.20). Setting ${ }^{8} \bar{a}_{m}^{B S}=m^{2}$, we then find that

$$
{ }^{\Delta}{\underset{m}{m}}_{\pi}^{(4) a s}=\frac{4 m}{\pi}\left(\frac{e^{2} q}{4 m^{2}}\right)^{m}, \quad m=1,2,3, \ldots .
$$

The rigorous asymptotic mathematical result is ${ }^{70}$

$$
{ }^{8} \Delta_{m}^{a s}=\frac{8}{[(m-1) !]^{2}}\left(\frac{q}{4}\right)^{m}, \quad m=1,2,3, \ldots .
$$

If we use de Moivre's form ${ }^{71}$ of Stirling's approximation

$$
(m-1) ! \sim(2 \pi)^{1 / 2} m^{m-1} e^{-m}, \quad m=1,2,3, \ldots,
$$

we see that ${ }^{8} \Delta{ }_{m}^{(4) a s}$ and ${ }^{8} \Delta{ }_{m}^{a s}$ are asymptotically equivalent [note that Eq. (3.44) is also known ${ }^{72}$ in the mathematical literature].

Finally we remark that the above equations suggest that the energy of the center of a narrow band or gap may actually be displaced from its true position by a large number of widths. A similar phenomenon occurs in the uniform semiclassical theory of resonances in molecular orbiting collisions. ${ }^{41,66}$

\section{E. Discussion}

The semiclassical expressions derived in Sec. III B all involve the two action integrals $\alpha, \pi \epsilon$, and their derivatives. For narrow energy bands, the uniform semiclassical explanation for the splitting of the energy levels is the conventional one of tunneling through a barrier, i.e., the coordinate $\theta$ is real, the wave number $k(\theta) d \theta$ takes pure imaginary values in the barrier region but $\pi \epsilon$ remains real. For energies above the barrier maxima, the energy gaps arise from above-barrier reflection. In the uniform semiclassical treatment this is taken into account by allowing $\theta$ to be complex so that we have two complex conjugate turning points, $k(\theta) d \theta$ is imaginary (along a Stokes' line) and $\pi \epsilon$ is again real. The primitive semiclassical theory as represented by the Bohr-Sommerfeld quantization formulas only gives the energy for the center of a band or gap and incorrectly predicts that each level is doubly degenerate.

We consider next the application of these results to the avoided crossing problem described by two of us and Noid. ${ }^{11}$ In that problem an avoided crossing of eigenvalues for a two dimensional coupled oscillator system was generated by $(3: 1)$ Fermi resonance and was analyzed in terms of a Schrödinger equation involving action angle variables. One of the actions-the total action-was a constant of the motion. There remained a one dimensional Schrödinger equation containing a "slow" angle variable which was equivalent to a Mathieu equation of fractional order. Each avoided crossing involved pairs of eigenvalues for which the $x$ and $y$ quantum numbers $n_{x}$ and $n_{y}$ respectively, satisfied $n=3 n_{x}+n_{y}+2$, where $n$ is an integer and $n_{x}, n_{y}=0,1,2, \ldots$. Thus, e.g., the $\left(n_{x}, n_{y}\right)$ states $(0,3)$ and $(1,0)$ underwent an avoided crossing as did the $(0,6),(2,0)$ pair, the $(0,9),(3,0)$ pair, the $(1,3),(2,0)$ pair, and so on. Each avoided crossing occurred at a different value of a perturbation parameter $\lambda$ in the Hamiltonian. The minimum splitting of the avoided crossing was given by $\left(a_{m}-b_{m}\right) / D$, where $D$ is a known constant, $a_{m}$ and $b_{m}$ are the eigenvalues of Mathieu's equation of integer order and $m=\left|n_{x}^{(1)}-n_{x}^{(2)}\right|$, i.e., the difference in the $n_{x}$ values of the pair of eigenstates undergoing the avoided crossing. Equations (3.44) or (3.45) show that for small $q$, this splitting de- 
creases rapidly with increasing $\left|n_{x}^{(1)}-n_{x}^{(2)}\right|$ in a sequence of pairs, such as $\left(n_{x}, 0\right),\left(0,3 n_{x}\right)$.

The above analysis corresponds semiclassically to a splitting at the avoided crossing due to an "over the barrier" reflection process. This barrier occurs in the angle variable and involves semiclassically a path between complex values of the angle variable but having a real (conjugate) action. When expressed in terms of the original Cartesian coordinates and momenta of the coupled oscillator Hamiltonian, it again corresponds, in the first approximation, to an over the barrier reflection. ${ }^{11}$ As noted in Ref. 11 , each of the primitive semiclassical states is associated with a particular trajectory and the two trajectories associated with the quantum mechanical avoided crossing can be connected to each other with the classically forbidden path just described.

Another view of a pair of trajectories connected by a classically forbidden path is that of Lawton and Child, ${ }^{73}$ who treated a pair of local mode vibrations of $\mathrm{H}_{2} \mathrm{O}$, and found that these local modes are each associated with a separate classical trajectory. This pair of trajectories was connected by a classically forbidden path which involved a tunneling in Cartesian coordinate space from the cusp of one caustic to a cusp of the other caustic. The splitting of the local mode energies was then calculated using the semiclassical formula for the splitting of the eigenvalues of a symmetric double well potential. ${ }^{73}$ This phenomenon has been termed "dynamical tunneling" by Davis and Heller ${ }^{34,35}$ in that there is no energy barrier between the two trajectories arising from the original potential surface, rather a (dynamical) barrier is generated by the existence of certain variables which are approximately constants of the motion. Although Lawton and Child calculated the splitting of the two states using an approximate cusp-to-cusp (double well) tunneling path, Sibert et al. ${ }^{12}$ were able to calculate the splitting using an over-thebarrier reflection model in which the barrier was along some angle coordinate. Thus it is quite possible that in our case also, a cusp-to-cusp (double well) tunneling path may be found to explain the splitting even though the approximate theory of Ref. 11 involves a classically forbidden over-thebarrier reflection path.

Finally we remark that the present semiclassical analysis for Mathieu functions of integer order is easily extended to functions of fractional order of the kind used in Ref. 11 by solving the general equation (2.33) with $\sigma \neq 0$. It is also straightforward to derive formulas corresponding to ${ }^{b} \Delta \underset{m}{(4)}$ and ${ }^{8}{ }_{m}^{(4)}$ for the case $\sigma \neq 0$.

\section{NUMERICAL CALCULATIONS AND RESULTS}

\section{A. Introduction}

The formulas we derived in Sec. III do not have error bounds associated with them. It is therefore important to carry out numerical computations to assess their validity. The results we present below are for $q=4$ since this case has also been investigated by Fröman ${ }^{26,27}$ using higher order phase integral techniques.

\section{B. Computational methods}

The complete ellipticintegrals $K(k)$ and $E(k)$ were evaluated by Gauss-Mehler quadrature. ${ }^{74}$ For $0<\epsilon<5.0$, $\arg \Gamma\left(\frac{1}{2}+i \epsilon\right)$ was calculated from the expansion ${ }^{75}$

$$
\arg \Gamma\left(\frac{1}{2}+i \epsilon\right)=\psi\left(\frac{1}{2}\right) \epsilon+\sum_{n=0}^{\infty}\left[\frac{\epsilon}{n+\frac{1}{2}}-\tan ^{-1}\left(\frac{\epsilon}{n+\frac{1}{2}}\right)\right]
$$

where $\psi\left(\frac{1}{2}\right)=-\gamma-2 \ln 2$ and $\gamma$ is Euler's constant. ${ }^{75}$ For $\epsilon>5.0$ we evaluated the quantum correction function $\phi(\epsilon)$ directly from its three term asymptotic expansion. ${ }^{41}$

Equation (3.10) shows that we must also calculate $\operatorname{Re} \psi\left(\frac{1}{2}+i \epsilon\right)$ in order to determine $d \phi / d a$. We used the following expansion ${ }^{75}$ :

TABLE I. Exact eigenvalues $a$ for the Mathieu equation with $q=4$. Approximate results are shown as ratios of the exact values. $a^{\text {sco }}$ is the uniform semiclassical eigenvalue from Eqs. (3.4) or (3.5). $a^{s c}$ is obtained from the same equations but with $\phi \equiv 0$. The quantity $\bar{a}$ is the average of the exact eigenvalues for narrow energy bands or narrow energy gaps. $\bar{a}^{B S \phi}$ is the Bohr-Sommerfeld semiclassical average energy calculated from Eq. (3.6) for narrow bands or Eq. (3.15) for narrow energy gaps. $\bar{a}^{B S}$ is obtained from the same equations but with $\phi \equiv 0$. The asymptotic average energies $\bar{a}^{a s}$ are given by Eq. (3.34) or (3.42). The dashed line indicates the top of the barrier at $2 q=8$.

\begin{tabular}{|c|c|c|c|c|c|c|c|c|c|}
\hline $\begin{array}{l}\text { Band } \\
\text { edge }\end{array}$ & $a$ & $a^{s c \phi} / a$ & $a^{s c} / a$ & $\bar{a}$ & $\bar{a}^{s c \phi} / \bar{a}$ & $\bar{a}^{x} / \bar{a}$ & $\bar{a}^{B S \phi} / \bar{a}$ & $\bar{a}^{B S} / \bar{a}$ & $\bar{a}^{a s} / \bar{a}$ \\
\hline$a_{0}$ & -4.28051882 & 0.981 & 0.967 & & \multirow{3}{*}{$\begin{array}{l}0.981 \\
1.034\end{array}$} & \multirow[b]{2}{*}{0.967} & \multirow[b]{2}{*}{0.981} & \multirow[b]{2}{*}{0.967} & \multirow[b]{2}{*}{0.937} \\
\hline $\begin{array}{l}b_{1} \\
a_{1}\end{array}$ & $\begin{array}{r}-4.25918290 \\
2.31800817\end{array}$ & $\begin{array}{l}0.981 \\
1.039\end{array}$ & $\begin{array}{l}0.967 \\
1.090\end{array}$ & \multirow{2}{*}{2.5324} & & & & & \\
\hline $\begin{array}{l}b_{2} \\
a_{2}\end{array}$ & $\begin{array}{r}2.74688103 \\
6.82907484 \\
\end{array}$ & $\begin{array}{l}1.029 \\
1.015 \\
\end{array}$ & $\begin{array}{l}1.085 \\
1.039\end{array}$ & & & 1.087 & 1.027 & 1.079 & 1.580 \\
\hline $\bar{b}_{3}$ & 9.26144613 & $\overline{1} . \overrightarrow{00} \overrightarrow{8}$ & 0.975 & \multirow{2}{*}{9.9662} & \multirow{2}{*}{1.008} & \multirow{2}{*}{0.983} & & & \\
\hline $\begin{array}{l}a_{3} \\
b_{4}\end{array}$ & $\begin{array}{l}10.67102710 \\
16.45203529\end{array}$ & $\begin{array}{l}1.008 \\
1.004\end{array}$ & $\begin{array}{l}0.991 \\
0.997\end{array}$ & & & & 1.023 & 0.999 & 0.992 \\
\hline $\begin{array}{l}a_{4} \\
b_{3}\end{array}$ & $\begin{array}{l}16.64981891 \\
25.33054487\end{array}$ & $\begin{array}{l}1.004 \\
1.002\end{array}$ & $\begin{array}{l}0.998 \\
0.999\end{array}$ & 16.5509 & 1.004 & 0.997 & 1.004 & 0.998 & 0.997 \\
\hline $\begin{array}{l}a_{5} \\
b_{6}\end{array}$ & $\begin{array}{l}25.34375763 \\
36.22941142\end{array}$ & $\begin{array}{l}1.002 \\
1.001\end{array}$ & $\begin{array}{l}0.999 \\
1.000\end{array}$ & 25.3372 & 1.002 & 0.999 & 1.002 & 0.999 & 0.999 \\
\hline $\begin{array}{l}a_{6} \\
b_{7}\end{array}$ & $\begin{array}{l}36.22995251 \\
49.16706763\end{array}$ & $\begin{array}{l}1.001 \\
1.001\end{array}$ & $\begin{array}{l}1.000 \\
1.000\end{array}$ & 36.2297 & 1.001 & 1.000 & 1.001 & 1.000 & 1.000 \\
\hline $\begin{array}{l}a_{7} \\
b_{8}\end{array}$ & $\begin{array}{l}49.16708282 \\
64.12715914\end{array}$ & $\begin{array}{l}1.001 \\
1.001\end{array}$ & $\begin{array}{l}1.000 \\
1.000\end{array}$ & \multirow{2}{*}{$\begin{array}{l}49.1671 \\
64.1272\end{array}$} & 1.001 & 1.000 & 1.001 & 1.000 & 1.000 \\
\hline$a_{8}$ & 64.12715945 & 1.001 & 1.000 & & 1.001 & 1.000 & 1.001 & 1.000 & 1.000 \\
\hline
\end{tabular}




$$
\operatorname{Re} \psi\left(\frac{1}{2}+i \epsilon\right)=\psi\left(\frac{1}{2}\right)+\epsilon^{2} \sum_{n=1}^{\infty} \frac{7 n^{2}+4 \epsilon^{2}}{n\left(n^{2}+\epsilon^{2}\right)\left(n^{2}+4 \epsilon^{2}\right)} .
$$

This expansion can be obtained from the duplication formula for the digamma function ${ }^{75}$

$$
\psi(2 z)=\frac{1}{2} \psi(z)+\frac{1}{2} \psi\left(z+\frac{1}{2}\right)+\ln 2,
$$

with $z=\frac{1}{2}+i \epsilon$, together with the result ${ }^{75}$

$$
\operatorname{Re} \psi(1+i y)=-\gamma+y^{2} \sum_{n=1}^{\infty} n^{-1}\left(n^{2}+y^{2}\right)^{-1}
$$

\section{Results}

Table I shows our results for the first 17 eigenvalues of the Mathieu equation when $q=4$. The accurate eigenvalues $a$ are from Ref. 76. Ten figure accuracy is necessary to calculate the widths $a_{m}-b_{m}$ of the small gaps. Notice that for $a_{8}-b_{8}$ we are only able to calculate the gap width to two figure accuracy.

The uniform semiclassical eigenvalues obtained from Eqs. (3.4) or (3.5) are denoted by $a^{s c \phi}$, or by $a^{s c}$ when we put $\phi \equiv 0$. The semiclassical expressions for the widths of the bands ${ }^{b} \Delta_{m}^{(i)}, i=1,2,3,4,5$ and the gaps ${ }^{g} \Delta_{m}^{(i)}, i=1,2,3,4$ require that we specify an energy for the center of a band or gap as appropriate. For $a, a^{s c \phi}, a^{s c}$ this energy was obtained by taking the mean of the neighboring eigenvalues. These averaged energies are denoted $\bar{a}, \bar{a}^{s c \phi}, \bar{a}^{s c}$, respectively. The average energy obtained from the Bohr-Sommerfeld quantization formulas (3.6) or (3.15) is denoted $\bar{a}^{B S \phi}$ or $\bar{a}^{B S}$ when $\phi \equiv 0$. Finally the asymptotic energies (3.34) or (3.42) are denoted $a^{a s}$. A superscript " $b$ " or " $g$ " is added when it is necessary to distinguish between a band and a gap.

Table I shows that generally all the semiclassical results agree well with the exact ones. In particular $a^{s c \phi}$ agrees with the exact $a$ to within $4 \%$, or much better than this. Notice

TABLE II. Exact and approximate widths of narrow energy bands for the Mathieu equation with $g=4$. The exact widths are denoted by ${ }^{b} \Delta_{m}$. Approximate results are shown as ratios of the accurate values. The numbers in parentheses are the power of ten by which the entry must be multiplied. The superscripts 1, 2, 3, 4, 5 refer to Eqs. (3.8), (3.11), (3.12), (3.14), (3.40), re-

\begin{tabular}{|c|c|c|c|c|c|}
\hline \multirow[b]{2}{*}{ Ratio } & \multicolumn{5}{|c|}{ Mean eigenvalue } \\
\hline & $\bar{a}_{m}$ & $\bar{a}_{m}^{s c \phi}$ & $\bar{a}_{m}^{s c}$ & $\bar{a}_{m}^{B S \phi}$ & $\bar{a}_{m}^{B S}$ \\
\hline & \multicolumn{2}{|c|}{$m=1$} & \multicolumn{3}{|c|}{${ }^{b} \Delta_{1}=b_{1}-a_{0}=2.1336(-2)$} \\
\hline${ }^{b} \Delta_{1}^{(1) / b} \|^{b}$ & 0.889 & 0.928 & 0.957 & 0.928 & 0.957 \\
\hline${ }^{b} \Delta_{1}^{(2)} / f^{b} \Delta_{1}$ & 0.889 & 0.928 & 0.957 & 0.928 & 0.957 \\
\hline${ }^{b} \Delta_{1}^{|3|} /{ }^{b} \Delta_{1}$ & 0.894 & 0.933 & 0.963 & 0.934 & 0.963 \\
\hline${ }^{b} \Delta_{1}^{(4) / b} \Delta_{1}$ & 0.894 & 0.933 & 0.963 & 0.934 & 0.963 \\
\hline \multirow[t]{3}{*}{${ }^{b} \Delta_{1}^{(s)} /{ }^{b} \Delta_{1}$} & 0.961 & 1.003 & 1.035 & 1.004 & 1.035 \\
\hline & \multicolumn{2}{|c|}{${ }^{b} \Delta_{1}^{a s} /{ }^{b} \Delta_{1}=1.135$} & \multicolumn{3}{|c|}{${ }^{b} \Delta_{1}^{(4) a s} /{ }^{b} \Delta_{1}=1.056$} \\
\hline & \multicolumn{2}{|c|}{$m=2$} & \multicolumn{3}{|c|}{${ }^{b} \Delta_{2}=b_{2}-a_{1}=4.2887(-1)$} \\
\hline${ }^{b} \Delta_{2}^{(1)} / /^{b} \Delta_{2}$ & 0.941 & 0.972 & 1.024 & 0.966 & 1.015 \\
\hline${ }^{b} \Delta_{2}^{(2)} /{ }^{b} \Delta_{2}$ & 0.944 & 0.976 & 1.028 & 0.969 & 1.020 \\
\hline${ }^{b} \Delta_{2}^{(3) / b} \Delta_{2}$ & 0.968 & 1.001 & 1.055 & 0.994 & 1.046 \\
\hline${ }^{b} \Delta_{2}^{(4) / b} \Delta_{2}$ & 0.971 & 1.005 & 1.059 & 0.998 & 1.051 \\
\hline \multirow[t]{2}{*}{${ }^{b} \Delta_{2}^{(5) / b} \Delta_{2}$} & 0.998 & 1.032 & 1.088 & 1.025 & 1.080 \\
\hline & \multicolumn{2}{|c|}{${ }^{b} \Delta_{2}^{a s} / b \Delta_{2}=1.80$} & \multicolumn{3}{|c|}{${ }^{b} \Delta_{2}^{(4) a s} /{ }^{b} \Delta_{2}=1.759$} \\
\hline
\end{tabular}
spectively, while ${ }^{b} \Delta_{m}^{(4) a s}$ and ${ }^{b} \Delta_{m}^{a s}$ are given by Eqs. (3.37) and (3.38), respectively. that $a^{s c \phi}$ is more accurate than $a^{s c}$ in the neighborhood of the barrier maximum, thereby illustrating the utility of the quantum correction function $\phi$. At higher values of $m, a^{s c}$ becomes more accurate than $a^{s c \phi}$ although the error in $a^{s c \phi}$ is only $0.1 \%$. This behavior is a consequence of the parabolic barrier comparison equation used for the calculation of $\phi$. The assumption of an approximately parabolic potential becomes less valid as the complex turning points move far into the complex $\theta$ plane. For the average energy of a gap all methods are in close agreement with the exact $\bar{a}$. For the case

TABLE III. Exact and approximate widths of narrow energy gaps for the Mathieu equation with $q=4$. The exact widths are denoted ${ }^{8} \Delta_{m}$. Approximate results are shown as ratios of the accurate values. The numbers in parentheses are the power of ten by which the entry must be multiplied. The

\begin{tabular}{|c|c|c|c|c|c|}
\hline \multirow[b]{2}{*}{ Ratio } & \multicolumn{5}{|c|}{ Mean eigenvalue } \\
\hline & $\bar{a}_{m}$ & $\bar{a}_{m}^{s c \phi}$ & $\bar{a}_{m}^{s c}$ & $\bar{a}_{m}^{B S \phi}$ & $\bar{a}_{m}^{B S}$ \\
\hline & \multicolumn{2}{|c|}{$m=3$} & \multicolumn{3}{|c|}{${ }^{8} \Delta_{3}=a_{3}-b_{3}=1.4096$} \\
\hline${ }^{g} \Delta_{3}^{(1) / g} \Delta_{3}$ & 1.008 & 0.987 & 1.052 & 0.949 & 1.010 \\
\hline${ }^{8} \Delta_{3}^{(2)} / g_{\Delta}$ & 1.077 & 1.051 & 1.134 & 1.005 & 1.080 \\
\hline${ }^{8} \Delta_{3}^{(3) / 8} \Delta_{3}$ & 1.037 & 1.018 & 1.075 & 0.983 & 1.039 \\
\hline \multirow[t]{3}{*}{${ }^{8} \Delta_{3}^{(4)} / 8 \Delta_{3}$} & 1.108 & 1.084 & 1.158 & 1.041 & 1.111 \\
\hline & \multicolumn{2}{|c|}{${ }^{8} \Delta_{3}^{a s} /{ }^{g} \Delta_{3}=1.419$} & \multicolumn{3}{|c|}{${ }^{8} \Delta_{3}^{(4) a s} /{ }^{8} \Delta_{3}=1.500$} \\
\hline & \multicolumn{2}{|c|}{$m=4$} & \multicolumn{3}{|c|}{${ }^{8} \Delta_{4}=a_{4}-b_{4}=1.9778(-1)$} \\
\hline${ }^{8} \Delta_{4}^{(1) / 8} \Delta_{4}$ & 1.033 & 1.012 & 1.047 & 1.011 & 1.046 \\
\hline${ }^{8} \Delta_{4}^{(2)} / 8 \Delta_{4}$ & 1.034 & 1.013 & 1.048 & 1.012 & 1.047 \\
\hline${ }^{8} \Delta_{4}^{(3)} / 8 \Delta_{4}$ & 1.048 & 1.026 & 1.062 & 1.025 & 1.061 \\
\hline \multirow[t]{3}{*}{${ }^{8} \Delta_{4}^{(4) / 8} \Delta_{4}$} & 1.048 & 1.027 & 1.063 & 1.026 & 1.062 \\
\hline & \multicolumn{2}{|c|}{${ }^{\mathrm{g}} \Delta_{4}^{a s} /{ }^{g} \Delta_{4}=1.124$} & \multicolumn{3}{|c|}{${ }^{8} \Delta_{4}^{(4 \mid a s} / g_{\Delta_{4}}=1.171$} \\
\hline & \multicolumn{2}{|c|}{$m=5$} & \multicolumn{3}{|c|}{${ }^{g} \Delta_{5}=a_{5}-b_{5}=1.3213(-2)$} \\
\hline${ }^{8} \Delta_{5}^{(1) / 8} \Delta_{5}$ & 1.030 & 1.013 & 1.034 & 1.013 & 1.035 \\
\hline${ }^{g} \Delta_{5}^{(2) / g} \Delta_{5}$ & 1.030 & 1.013 & 1.034 & 1.013 & 1.035 \\
\hline${ }^{8} \Delta_{5}^{(3)} / 8 \Delta_{5}$ & 1.034 & 1.017 & 1.038 & 1.017 & 1.038 \\
\hline \multirow[t]{3}{*}{${ }^{8} \Delta_{3}^{(4) / 8} \Delta_{5}$} & 1.034 & 1.017 & 1.038 & 1.017 & 1.038 \\
\hline & \multicolumn{2}{|c|}{${ }^{g} \Delta_{s}^{a s} / g \Delta_{5}=1.051$} & \multicolumn{3}{|c|}{${ }^{8} \Delta{\stackrel{(4) a s}{(g} /{ }^{g}}_{\Delta}=1.087$} \\
\hline & \multicolumn{2}{|c|}{$m=6$} & \multicolumn{3}{|c|}{${ }^{8} \Delta_{6}=a_{6}-b_{6}=5.4109(-4)$} \\
\hline${ }^{8} \Delta_{6}^{(1 !) / 8} \Delta_{6}$ & 1.026 & 1.013 & 1.028 & 1.013 & 1.028 \\
\hline $8_{4}^{(2) / 8} \Delta_{6}$ & 1.026 & 1.013 & 1.028 & 1.013 & 1.028 \\
\hline${ }^{8} \Delta_{6}^{(3)} / /^{8} \Delta_{6}$ & 1.028 & 1.014 & 1.030 & 1.014 & 1.030 \\
\hline \multirow[t]{3}{*}{${ }^{8} \Delta_{6}^{(4) / 8} \Delta_{6}$} & 1.028 & 1.014 & 1.030 & 1.014 & 1.030 \\
\hline & \multicolumn{2}{|c|}{${ }^{8} \Delta_{6}^{a s} /{ }^{g} \Delta_{6}=1.027$} & \multicolumn{3}{|c|}{${ }^{8} \Delta_{6}^{(4) a s / g} \Delta_{6}=1.056$} \\
\hline & \multicolumn{2}{|c|}{$m=7$} & \multicolumn{3}{|c|}{${ }^{\mathrm{g}} \Delta_{7}=a_{7}-b_{7}=1.519(-5)$} \\
\hline$g_{\Delta}^{(1) / 8} \Delta_{7}$ & 1.023 & 1.012 & 1.024 & 1.012 & 1.024 \\
\hline${ }^{8} \Delta_{7}^{(2)} / 8 \Delta_{7}$ & 1.023 & 1.012 & 1.024 & 1.012 & 1.024 \\
\hline${ }^{8} \Delta_{7}^{(3) / 8} \Delta_{7}$ & 1.024 & 1.013 & 1.025 & 1.013 & 1.025 \\
\hline \multirow[t]{3}{*}{${ }^{8} \Delta_{7}^{(4)} /{ }^{8} \Delta_{7}$} & 1.024 & 1.013 & 1.025 & 1.013 & 1.025 \\
\hline & \multicolumn{2}{|c|}{${ }^{8} \Delta{ }_{7}^{a s} /{ }^{8} \Delta_{7}=1.016$} & \multicolumn{3}{|c|}{${ }^{8} \Delta \stackrel{(4) a s}{7} / 8^{8} \Delta_{7}=1.040$} \\
\hline & & & ${ }^{8} \Delta_{8}=$ & $-b_{8}=$ & $1(-7)$ \\
\hline${ }^{8} \Delta_{8}^{(1) / 8} \Delta_{8}$ & 1.026 & 1.017 & 1.027 & 1.017 & 1.027 \\
\hline${ }^{8} \Delta_{8}^{(2) /{ }^{8} \Delta_{8}}$ & 1.026 & 1.017 & 1.027 & 1.017 & 1.027 \\
\hline${ }^{8} \Delta_{8}^{(3) / 8} \Delta_{8}$ & 1.027 & 1.017 & 1.027 & 1.017 & 1.027 \\
\hline${ }^{8} \Delta_{8}^{|4| / 8} \Delta_{8}$ & 1.027 & 1.017 & 1.027 & 1.017 & 1.027 \\
\hline & ${ }^{8} \Delta_{\mathrm{B}}^{a s} / g_{\Delta}$ & 1.016 & & ${ }^{8} / 8 \Delta_{8}$ & \\
\hline
\end{tabular}
superscripts 1, 2, 3, 4 refer to Eqs. (3.17), (3.18), (3.19), (3.20), respectively while ${ }^{8} \Delta_{m}^{(4) a s}$ and ${ }^{8} \Delta_{m}^{a s}$ are given by Eqs. (3.44) and (3.45), respectively. 
of narrow bands the discrepancies are greater especially for the bandwidth $b_{2}-a_{1}$ as given by $\vec{a}^{a s}$.

Tables II and III show the exact and approximate widths for narrow bands and gaps, respectively. For energies well removed from the barrier maximum we have for a given $i,{ }^{b} \Delta_{m}^{(i)}\left(\bar{a}_{m}^{s c \phi}\right) \approx{ }^{b} \Delta_{m}^{(i)}\left(\bar{a}_{m}^{B S \phi}\right)$ and ${ }^{b} \Delta_{m}^{(i)}\left(\bar{a}_{m}^{s c}\right) \approx^{b} \Delta_{m}^{(i)}\left(\bar{a}_{m}^{B S}\right)$ and similarly for the gap widths. This behavior is a consequence of the fact that $\bar{a}^{s c \phi} \approx \bar{a}^{B S \phi}$ and $\bar{a}^{s c} \approx \bar{a}^{B S}$ (see Table I). In general the $\Delta_{m}^{(i)}$ agree to within $5 \%$ of the exact results although occasionally the error is larger, e.g., $11 \%$ for ${ }^{b} \Delta_{1}^{(1)}\left(\bar{a}_{1}\right)$ or for $m=3$. An important advantage of the $\Delta_{m}^{(i)}$ is that the widths of narrow bands and gaps are given directly and do not involve taking the difference of two nearly identical numbers, as is necessary for the exact numerical results or if the full semiclassical formulas (3.4) and (3.5) are used. The results in Tables II and III suggest that the semiclassical $\Delta_{m}^{(i)}$ can be used to calculate reliably very narrow band and gap widths for cases where accurate exact or full semiclassical values are not available. Incidently, for potentials which give rise to narrow bands or gaps lying close to a barrier maximum, it is essential to use the semiclassical width formulas (3.8), (3.11) or (3.17), (3.18) which include $d \phi / d E$, in order to cancel the infinite divergence contained in $d \alpha / d E$ at the barrier maximum.

Sometimes the trends in the results differ from those expected from the derivation presented in Sec. III B. It is likely that this arises in part from cancellation of errors, since the same effect has been observed in semiclassical calculations of resonance energies and widths for orbiting resonances. ${ }^{41,66}$ To better understand the errors in the semiclassical results, it would be necessary to calculate eigenvalues and widths over an extended range of $q$ values. Finally we note that Tables II and III show that the asymptotic widths $\Delta_{m}^{a s}$ and $\Delta_{m}^{(4) a s}$ are generally less accurate than the semiclassical widths $\Delta_{m}^{(i)}$.

\section{SUMMARY AND CONCLUSIONS}

In this article we have derived a uniform semiclassical expression for the eigenvalues of a one dimensional Schrödinger equation with a periodic potential but with nonperiodic boundary conditions. The semiclassical analysis used parabolic connection formulas for a barrier and the resulting formulas are valid for real or complex classical turning points as the energy passes through a barrier maximum. For a potential with periodicity $2 \pi / m$ our quantization formula generalizes and unifies previous formulas in the literature.

A detailed application was made to Mathieu functions of integer order. Similar detailed treatments could be given for more complicated problems such as Mathieu functions of fractional order or for potentials with more than two barriers in $(0,2 \pi]$. We derived semiclassical formulas for the widths of the energy bands and energy gaps of the periodic Mathieu equation when these quantities are small. All the action integrals appearing in the semiclassical formulas can be expressed in terms of complete elliptic integrals thereby letting us exploit the considerable body of theory that exists for these integrals. We showed that the semiclassical formulas are asymptotically equivalent to results in the mathematical literature. This gives a physical interpretation to the mathematical results which apparently has not been done before.
Our results for the splitting of the eigenvalues provide an example of dynamical tunneling when applied to the avoided crossing problem of Ref. 11 . However, in the present case the splitting arises from an above barrier reflection process rather than from tunneling below the barrier as occurs for a symmetric double well potential.

\section{ACKNOWLEDGMENTS}

Support of this research by the National Science Foundation (U.S.A.) and the Science and Engineering Research Council (U.K.) is gratefully acknowledged. We are grateful to NATO for a Senior Scientist Award to J.N.L.C.

${ }^{1}$ H. D. Koenig, Phys. Rev. 44, 657 (1933).

${ }^{2}$ J. J. Stoker, Nonlinear Vibrations in Mechanical and Electrical Systems (Interscience, New York, 1950), Chap. VI.

${ }^{3} \mathrm{H}$. Jones, The Theory of Brillouin Zones and Electronic States in Crystals (North-Holland, Amsterdam, 1960), Chap. 1.

${ }^{4}$ F. M. Arscott, Periodic Differential Equations. An Introduction to Mathieu, Lamé, and Allied Functions (MacMillan, New York, 1964).

${ }^{5}$ H. Hochstadt, The Functions of Mathematical Physics (Wiley-Interscience, New York, 1971), Chap. 9

${ }^{6}$ M. S. P. Eastham, The Spectral Theory of Periodic Differential Equations (Scottish Academic, Edinburgh, 1973).

${ }^{7}$ W. Magnus and S. Winkler, Hill's Equation (Dover, New York, 1979).

${ }^{8}$ D. W. Noid, M. L. Koszykowski, and R. A. Marcus, Annu. Rev. Phys. Chem. 32, 267 (1981).

${ }^{9}$ D. W. Noid, M. L. Koszykowski, and R. A. Marcus, J. Chem. Phys. 78, 4018 (1983).

${ }^{10}$ R. A. Marcus, Discuss. Faraday Chem. Soc. 75, 103 (1983).

${ }^{11}$ T. Uzer, D. W. Noid, and R. A. Marcus, J. Chem. Phys. 79, 4412 (1983).

${ }^{12}$ E. L. Sibert III, J. T. Hynes, and W. P. Reinhardt, J. Chem. Phys. 77, 3595 (1982).

${ }^{13}$ C. Jaffé and W. P. Reinhardt, J. Chem. Phys. 77, 5191 (1982).

${ }^{14}$ H. H. Nielsen, Phys. Rev. 40, 445 (1932).

${ }^{15}$ J. S. Koehler and D. M. Dennison, Phys. Rev. 57, 1006 (1940); K. T. Hecht and D. M. Dennison, J. Chem. Phys. 26, 31 (1957).

${ }^{16}$ C. C. Lin and J. D. Swalen, Rev. Mod. Phys. 31, 841 (1959).

${ }^{17} \mathrm{C}$. H. Townes and A. L. Schawlow, Microwave Spectroscopy (McGrawHill, New York, 1955), Chap. 12.

${ }^{18}$ J. E. Wollrab, Rotational Spectra and Molecular Structure (Academic, New York, 1967), Chap. 6

${ }^{19}$ S. P. Karkach, V. I. Osherov, and V. G. Ushakov, Zh. Eksp. Teor. Fiz. 68, 493 (1975) [Sov. Phys. JETP 41, 241 (1975)].

${ }^{20}$ S. P. Karkach and V. I. Osherov, Mol. Phys. 36, 1069 (1978).

${ }^{21}$ S. C. Miller Jr. and R. H. Good Jr., Phys. Rev, 91, 174 (1953)

${ }^{22} J$. N. L. Connor, Mol. Phys. 15, 37 (1968).

${ }^{23}$ J. N. L. Connor, in Semiclassical Methods in Molecular Scattering and Spectroscopy, Proceedings of the NATO Advanced Study Institute held in Cambridge, England in September 1979, edited by M. S. Child (Reidel, Dordrecht, 1980), pp. 45-107.

${ }^{24}$ W. H. Miller, J. Chem. Phys. 48, 1651 (1968).

${ }^{25}$ P. Pajunen, J. Mol. Spectrosc. 88, 64 (1981); M. S. Child, ibid. 53, 280 (1974). See also M. S. Child, Proc. R. Soc. London Ser. A 292, 272 (1966); Discuss. Faraday Soc. 44, 68 (1967); pp. 127-154 of Ref. 23.

${ }^{26}$ N. Fröman, J. Phys. A 12, 2355 (1979).

${ }^{27}$ N. Fröman, Phys. Rev. D 23, 1756 (1981).

${ }^{28}$ N. Fröman and P. O. Fröman, J. Math. Phys. 221190 (1981).

${ }^{29}$ N. W. McLachlan, Theory and Application of Mathieu Functions (Dover, New York, 1964).

${ }^{30} \mathrm{R}$. Campbell, Théorie Générale de l'Équation de Mathieu et de Quelques autres Équations Differentielles de la Mécanique, préface du J. Pérès (Masson et Cie, Paris, 1955).

${ }^{31}$ J. Meixner and F. W. Schäfke, Mathieusche Funktionen und Sphäroidfunktionen mit Anwendungen auf Physikalische und Technische Probleme (Springer, Berlin, 1954). For Errata see pp. 117-119 of Ref. 32.

32J. Meixner, F. W. Schäfke, and G. Wolf, Mathieu Functions and Spheriodal Functions and their Mathematical Foundations: Further studies, Lect. Notes Math. No. 837 (1980). 
${ }^{33} \mathrm{G}$. Blanch, in Handbook of Mathematical Functions with Formulas, Graphs and Mathematical Tables, edited by M. Abramowitz and I. A. Stegun, Natl. Bur. Stand. Applied Math. Series No. 55 (U.S. Dept. of Commerce, Washington, D. C., 1972), Chap. 20.

${ }^{34}$ M. J. Davis and E. J. Heller, J. Chem. Phys. 75, 246 (1981).

${ }^{35}$ E. J. Heller and M. J. Davis, J. Phys. Chem. 85, 307 (1981); E. J. Heller, in Potential Energy Surfaces and Dynamics Calculations for Chemical Reactions and Molecular Energy Transfer, edited by D. G. Truhlar (Plenum, New York, 1981), p. 103.

${ }^{36}$ J. N. L. Connor, D. Phil. thesis, University of Oxford, Oxford, England, 1969.

${ }^{37}$ E. Merzbacher, Quantum Mechanics (Wiley, New York, 1961), Chaps.6 and 7.

${ }^{38}$ J. N. L. Connor, Mol. Phys. 15, 621 (1968).

${ }^{39}$ S. M. Colwell, N. C. Handy, and W. H. Miller, J. Chem. Phys. 68, 745 (1978); Lect. Notes Phys. 93, 299 (1979).

${ }^{40}$ N. L. Balazs, Ann. Phys. (N.Y.) 53, 421 (1969); M. J. Richardson, Am. J. Phys. 39, 560 (1971).

${ }^{41}$ J. N. L. Connor and A. D. Smith, Mol. Phys. 43, 397 (1981).

${ }^{42}$ J. Luppi and P. Pajunen, J. Chem. Phys. 78, 4451 (1983).

${ }^{43}$ J. N. L. Connor, Chem. Phys. Lett. 4, 419 (1969).

${ }^{44}$ E. U. Condon, Phys. Rev. 31, 891 (1928); T. Pradhan and A. V. Khare, Am. J. Phys. 41, 59 (1973); H. Neuberger, Phys. Rev. D 17, 498 (1978); P. Garbaczewski, Phys. Lett. A 71, 9 (1979); R. Aldrovandi and R. L. Ferreira, Am. J. Phys. 48, 660 (1980); R. N. Kesarwani and Y. P. Varshni, J. Math. Phys. 23, 92 (1982); A-M. M. Abdel-Rahman, Am. J. Phys. 51, 721 (1983); D. Kiang, ibid. 46, 1188 (1978).

${ }^{45}$ E. Thiele and D. J. Wilson, J. Chem. Phys. 35, 1256(1961); E. J. Heller, E. B. Stechel, and M. J. Davis, ibid. 73, 4720 (1980).

${ }^{46}$ J. C. Slater, Phys. Rev. 87, 807 (1952); E. R. Floyd, J. Math. Phys. 17, 880 (1976); 20, 83 (1979); B. F. Buxton and M. V. Berry, Philos. Trans. R. Soc. London Ser. A 282, 485 (1976); S. Y. Lee and N. Takigawa, Nucl. Phys. A 308, 189 (1978).

${ }^{47}$ L. Pauling, Phys. Rev. 36, 430 (1930).

${ }^{48}$ A. Khare, Lett. Nuovo Cimento 18, 346 (1977); N. Gupta and B. Sutherland, Phys. Rev. A 14, 1790 (1976); M. Stone and J. Reeve, Phys. Rev. D 18, 4746(1978); M. Asorey, J. G. Esteve, and A. F. Pacheco, ibid. 27, 1852 (1983).

${ }^{49}$ R. M. DeLeonardis and S. E. Trullinger, Phys. Rev. A 20, 2603 (1979).

${ }^{50}$ G. M. Zaslavskiĭ and N. N. Filonenko, Zh. Eksp. Teor. Fiz. 54, 1590 (1968) [Sov. Phys. JETP 25, 851 (1968)]; N. B. Narozhnyl and A. I. Nikishov, ibid. 65, 862 (1973) [Sov. Phys. JETP 38, 427 (1974)]; N. L. Manakov, L. P. Rapoport, and A. G. Fainshtein, Teor. Mat. Fiz. 30, 395 (1977) [Theo. Math. Phys. 30, 255 (1977)]; B. I. Meerson, E. A. Oks, and P. V. Sasorov, J. Phys. B 15, 3599 (1982); J. Bergou and S. Varró, J. Phys. A 13, 3553 (1981); K. Druhl and J. K. Mclver, J. Math. Phys. 24, 705 (1983); V. S. Letokhov and V. G. Minogin, J. Opt. Soc. Am. 69, 413 (1979); Phys. Rep. 73, 1 (1981).

${ }^{51}$ M. A. Hooshyar and M. Razavy, Nuovo Cimento B 75, 65 (1983); J. G. Leopold, I. C. Percival, and D. Richards, J. Phys. A 15, 805 (1982); P. A. Braun, Teor. Mat. Fiz. 37, 355 (1978) [Theo. Math. Phys. 37, 1070 (1978)].
${ }^{52}$ Problems in Quantum Mechanics, 3rd ed., edited by D. Ter Haar (Pion, London, 1975), pp. 144-147.

${ }^{53}$ A. M. Dykhne, Zh. Eksp. Teor. Fiz. 40, 1423 (1961) [Sov. Phys. JETP 13, 999 (1961)]. See also S. G. Simonyan, Diff. Uravn. 6, 1265 (1970) [Diff. Equat. 6, 965 (1970)].

${ }^{54}$ E. Trubowitz, Comm. Pure Appl. Math. 30, 321 (1977).

${ }^{55}$ P. F. Byrd and M. D. Friedman, Handbook of Elliptic Integrals for Engineers and Scientists, 2nd ed. (Springer, New York, 1971).

${ }^{56} \mathrm{~L}$. M. Milne-Thomson, in Ref. 33, Chap. 17.

${ }^{57}$ E. L. Ince, Proc. R. Soc. Edinburgh, 46, 20 (1925).

${ }^{58}$ See Ref. 29 , p. 40 , Fig. 8 or Ref. 33, p. 724, Fig. 20.1.

${ }^{59}$ H. Jeffreys, Proc. London Math. Soc. 23, 437 (1924) [reprinted in Collected Papers of Sir Harold Jeffreys on Geophysics and Other Sciences, edited by Sir Harold Jeffreys and B. Swirles (Lady Jeffreys) (Gordon and Breach, London, 1977), Vol. 6 p. 31; E. L. Ince, J. London Math. Soc. 2, 46 (1926); Proc. R. Soc. Edinburgh 46, 316 (1926); S. Goldstein, Trans. Cambridge Philos. Soc. 23, 303 (1927).

${ }^{60}$ Reference 29, p. 232, Eq. (1); Ref. 30, p. 112; Ref. 33, p. 726, Eq. (20.2.30).

${ }^{61}$ Reference 31, p. 139.

${ }^{62}$ Reference 33, p. 727, Eq. (20.2.31).

${ }^{63}$ S. Goldstein, Proc. R. Soc. Edinburgh 49, 210 (1929).

${ }^{64}$ Reference 29, p. 50, Sec. 3.43, Eq. (1). Reference 30, p. 120, Eq. (20).

${ }^{65}$ Sir H. Jeffreys and B. Swirles (Lady Jeffreys), Methods of Mathematical Physics, 3rd ed. (Cambridge University, Cambridge, 1962), p. 467, Eq. (9).

${ }^{66}$ J. N. L. Connor and A. D. Smith, Mol. Phys. 45, 149 (1982).

${ }^{67}$ E. M. Harrell, Ann. Phys. (N.Y.) 119, 351 (1979); T. P. Grozdanov and S. Yu Slavyanov, J. Phys. A 14, 1513 (1981).

${ }^{68}$ Reference 5, p. 307.

${ }^{69}$ Reference 29, p. 17, Eq. (14). Reference 33, p. 724, Eq. (20.2.26).

${ }^{70}$ M. Bell, Proc. Glasgow Math. Assoc. 3, 132 (1957); D. M. Levy and J. B. Keller, Comm. Pure Appl. Math. 16, 469 (1963); H. Hochstadt, ibid. 17, 251 (1964); V. M. Frolov, Diff. Uravn. 18, 1363 (1982) [Diff. Equat. 18, 959 (1982)]; J. Avron and B. Simon, Ann. Phys. (N.Y.) 134, 76 (1981).

${ }^{71}$ E. T. Copson, Asymptotic Expansions (Cambridge University, Cambridge, 1965), p. 57.

${ }^{72}$ E. M. Harrell II, in Contributions to Analysis and Geometry, edited by D. N. Clark, G. Pecelli, and R. Sacksteder (Johns Hopkins University, Baltimore, 1981), p. 139; C. Hunter and B. Guerrieri, Stud. Appl. Math. 64, 113 (1981); C. Hunter, in Spectral Theory of Differential Operators, edited by I. W. Knowles and R. T. Lewis, Proceedings of the Conference held at the University of Alabama in Birmingham, Birmingham, Alabama, U.S.A., March 26-28, 1981 (North-Holland, Amsterdam, 1981), p. 233.

${ }^{73}$ R. T. Lawton and M. S. Child, Mol. Phys. 37, 1799 (1979); 44, 709 (1981). Note that Eq. (8) in the second paper for the splitting of the semiclassical eigenvalues of a symmetric double well potential is in error by a factor of 2 (see Ref. 52 for the correct formula).

${ }^{74} \mathrm{P}$. J. Davis and I. Polonsky, in Ref. 33, p. 889, item 25.4.38.

${ }^{75}$ P. J. Davis, in Ref. 33, Chap. 6, items 6.1.27, 6.3.3, 6.3.8, 6.3.17.

${ }^{76}$ Tables Relating to Mathieu Functions, Characteristic Values, Coefficients, and Joining Factors, Natl. Bur. Stand. Appl. Math. Series No. 59, 2nd ed. (U.S. GPO, Washington, D.C., 1967). 